\title{
Millisecond-scale differences in neural activity in auditory cortex can drive decisions
}

\author{
Yang Yang ${ }^{1,2}$, Michael R DeWeese ${ }^{1,3}$, Gonzalo Otazu ${ }^{1}$, and Anthony M Zador ${ }^{1}$ \\ ${ }^{1}$ Cold Spring Harbor Laboratory, 1 Bungtown Road, Cold Spring Harbor, NY 11724 \\ ${ }^{2}$ Program in Neuroscience, Stony Brook University, NY 11794 \\ ${ }^{3}$ Physics Department and Helen Wills Neuroscience Institute, UC Berkeley, CA 94720
}

\begin{abstract}
Neurons in the auditory cortex can lock with millisecond precision to the fine timing of acoustic stimuli, but it is not known whether this precise spike timing can be used to guide decisions. We used chronically implanted microelectrode pairs to stimulate neurons in the rat auditory cortex directly. Here we demonstrate that rats can exploit differences in the timing of cortical activity as short as three milliseconds to guide decisions.
\end{abstract}

Animals can detect the fine timing of some stimuli. For example, interaural time differences of less than one millisecond are used for the spatial localization of sound ${ }^{1}$. It is also clear that cortical neurons can lock with millisecond precision to the fine timing of some stimuli in the auditory cortex ${ }^{2,3}$, the visual cortex ${ }^{4}$, somatosensory cortex ${ }^{5,6}$ and in vitro ${ }^{7}$. Furthermore, spike generation in the auditory cortex is controlled by a stereotyped and precisely timed sequence of excitatory input followed approximately three milliseconds later by inhibitory input ${ }^{8}$. However, although it has recently been established that even a few cortical spikes are sufficient to drive decisions ${ }^{9,}{ }^{10}$, it has been difficult to establish whether the fine timing of cortical activity can suffice.

We therefore set out to probe the precision with which the fine timing of neural activity in the auditory cortex could guide behaviour in the rat. For the spatial localization of sound, the relevant sub-millisecond interaural time difference cues are extracted by specialized subcortical structures. To ensure that we were probing cortical rather than subcortical mechanisms, we bypassed subcortical auditory pathways and trained animals to respond to direct intracortical electrical stimulation. We used transient biphasic current trains delivered via two chronically implanted intracortical microelectrodes ${ }^{11,12}$ to stimulate two populations of neurons in primary auditory cortex (area A1; Fig. 1a). We designed the stimulation patterns so that the only cue available to guide behaviour was the relative timing of the activity elicited in the two cortical populations.

Users may view, print, copy, and download text and data-mine the content in such documents, for the purposes of academic research, subject always to the full Conditions of use:http://www.nature.com/authors/editorial_policies/license.html\#terms 
We first trained adult male Long Evans rats to perform a simple auditory two alternative choice task $(2-\mathrm{AC})^{13}$. The animal initiated a trial by inserting its nose into the centre port of a three port operant chamber, triggering one of two acoustic stimuli. These stimuli indicated whether the left or right goal port would be rewarded with water (Fig. 1a; for details, see Supplemental material). Chance performance was 50\%. After animals reached criterion performance (> 90\%), we implanted electrodes at two sites (A and B; Fig. 1a) about $1.1 \mathrm{~mm}$ apart along the rostro-caudal axis in the rat's left primary auditory cortex (Fig. S3). We then substituted electrical stimulation $(<30 \mu \mathrm{A})$ through these electrodes for the acoustic cue on the 2-AC task. Stimulus 1 (associated with the left reward port) consisted of simultaneous (AB) stimulation of the two intracortical sites, whereas stimulus 2 (associated with the right reward port) consisted of sequential (B-ISI-A) stimulation of the two sites. The two stimuli were separated by a variable interstimulus interval (ISI) which ranged from 1 to $100 \mathrm{~ms}$. After animals reached criterion performance for long ISIs (100 or $35 \mathrm{~ms}$ ), we reduced the ISI to probe the psychophysical limit for discrimination in the timing of the activity of the two cortical populations.

Twenty-six rats successfully learned the easy discrimination (A vs. B) and were trained on more challenging tasks. The training history of one subject is illustrated in Fig. 1b. Like most subjects, it rapidly learned to perform the discrimination for long ISIs (24/26 subjects trained on ISI=100 or ISI=35 ms performed above chance, $\mathrm{p}<0.01$; see Fig. $1 \mathrm{c}$ and Supplemental material). Surprisingly, this subject also performed above chance $(\mathrm{p}<0.01)$ when challenged with the finer temporal discriminations of ISI $=5$ and even ISI $=3 \mathrm{~ms}$, but not ISI=1 ms. Over the population, most subjects (10/15) challenged with ISI $=5 \mathrm{~ms}$ performed above chance for 1 or more sessions, each session containing 100-300 trials ( $<<0.01$; Fig. 1c); in some sessions performance exceeded $90 \%$ even at this short interval (Fig. 1d). Two subjects (Fig. 1b and Fig. S2-o) performed above chance even for ISI=3ms, but none of four subjects trained on ISI=1ms performed above chance. Performance declined with task difficulty both among (Fig. 1c) and within (Fig. 1e) subjects, but performance was variable; differences in performance could also be due to variability in the location of the electrodes, the effectiveness of the electrical stimulation, or other experimental factors.

Our results demonstrate that even fine differences as short as three milliseconds in the timing of artificially induced neuronal activity in the auditory cortex can be used to guide behaviour. Although artificial cortical microstimulation can be perceptually indistinguishable from natural stimulation ${ }^{14}$, this need not always be the case; our results do not reveal the conditions under which such fine temporal differences are important for the readout of acoustically-evoked (i.e. natural) stimuli. Nevertheless, the ability of the animal to read out such subtle differences in timing raises the possibility that for some stimuli, the timing of cortical spikes can be behaviourally relevant.

Our experiments were conducted in the primary auditory cortex. Experiments in the somatosensory cortex have failed to find a correlation between spike timing and behaviour ${ }^{15}$. Audition is often considered to be a "fast" modality, and it is clearly one in which subtle differences in temporal structure can be behaviourally relevant. However, no special biophysical or circuit mechanisms need be posited to account for our result; many 
simple candidate neural circuits could mediate the readout of the fine timing differences we have described. Indeed, in auditory cortex the stereotyped sequence of excitatory activity followed $3 \mathrm{~ms}$ later by inhibitory activity ${ }^{8}$ suggests one possible mechanism for the present results. Further experiments will be needed to resolve whether the capacity to exploit fine temporal differences is unique to the auditory cortex, or if it is a general strategy for cortical function (but see $\operatorname{ref}^{15}$ ).

\section{Supplementary Material}

Refer to Web version on PubMed Central for supplementary material.

\section{References}

1. Harper NS, McAlpine D. Optimal neural population coding of an auditory spatial cue. Nature. 2004; 430:682-6. [PubMed: 15295602]

2. Heil P. Auditory cortical onset responses revisited. I. First-spike timing. J Neurophysiol. 1997; 77:2616-41. [PubMed: 9163380]

3. DeWeese MR, Wehr M, Zador AM. Binary spiking in auditory cortex. J Neurosci. 2003; 23:7940-9. [PubMed: 12944525]

4. Buracas GT, Zador AM, DeWeese MR, Albright TD. Efficient discrimination of temporal patterns by motion-sensitive neurons in primate visual cortex. Neuron. 1998; 20:959-69. [PubMed: 9620700]

5. Mountcastle VB, Talbot WH, Sakata H, Hyvarinen J. Cortical neuronal mechanisms in fluttervibration studied in unanesthetized monkeys. Neuronal periodicity and frequency discrimination. $\mathrm{J}$ Neurophysiol. 1969; 32:452-84. [PubMed: 4977839]

6. Panzeri S, Petersen RS, Schultz SR, Lebedev M, Diamond ME. The role of spike timing in the coding of stimulus location in rat somatosensory cortex. Neuron. 2001; 29:769-77. [PubMed: 11301035]

7. Mainen ZF, Sejnowski TJ. Reliability of spike timing in neocortical neurons. Science. 1995; 268:1503-6. [PubMed: 7770778]

8. Wehr M, Zador AM. Balanced inhibition underlies tuning and sharpens spike timing in auditory cortex. Nature. 2003; 426:442-6. [PubMed: 14647382]

9. Houweling AR, Brecht M. Behavioural report of single neuron stimulation in somatosensory cortex. Nature. 2008; 451:65-8. [PubMed: 18094684]

10. Huber D, et al. Sparse optical microstimulation in barrel cortex drives learned behaviour in freely moving mice. Nature. 2008; 451:61-4. [PubMed: 18094685]

11. Murphey DK, Maunsell JH. Behavioral detection of electrical microstimulation in different cortical visual areas. Curr Biol. 2007; 17:862-7. [PubMed: 17462895]

12. Salzman CD, Britten KH, Newsome WT. Cortical microstimulation influences perceptual judgements of motion direction. Nature. 1990; 346:174-7. [PubMed: 2366872]

13. Uchida N, Mainen ZF. Speed and accuracy of olfactory discrimination in the rat. Nat Neurosci. 2003; 6:1224-9. [PubMed: 14566341]

14. Romo R, Hernandez A, Zainos A, Salinas E. Somatosensory discrimination based on cortical microstimulation. Nature. 1998; 392:387-90. [PubMed: 9537321]

15. Luna R, Hernandez A, Brody CD, Romo R. Neural codes for perceptual discrimination in primary somatosensory cortex. Nat Neurosci. 2005; 8:1210-9. [PubMed: 16056223] 
a
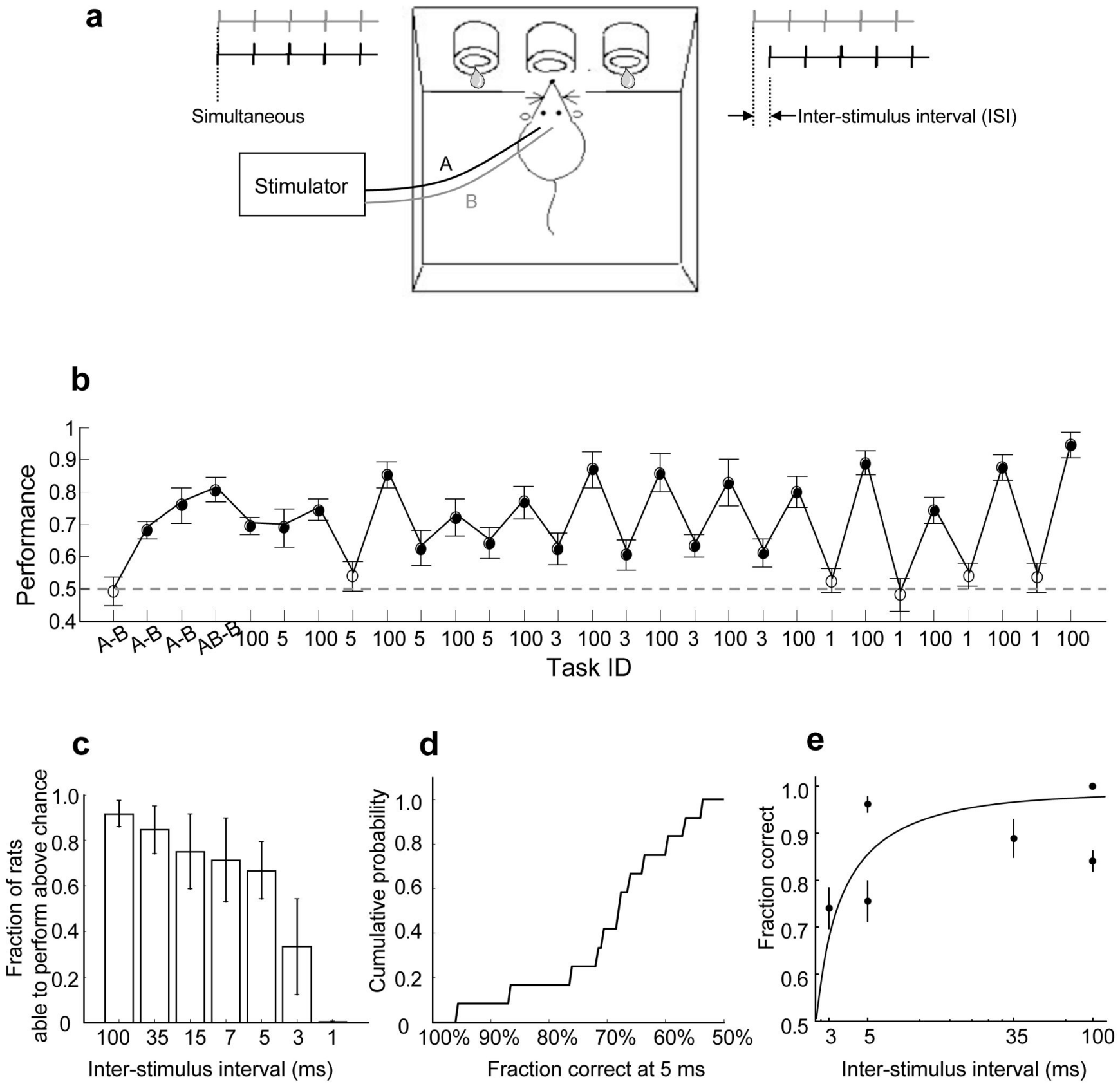

Figure 1. Finely timed cortical microstimulation can drive behaviour

(A) Task design. Animals were water deprived under a protocol approved by the Cold Spring Harbor Laboratory Animal Committee. Each stimulus consisted of a $50 \mathrm{~Hz}$ train of 5 biphasic cathode-leading current pulses. In one animal (Fig. S2-a) we used a symmetric discrimination A-ISI-B vs. B-ISI-A, rather than AB vs. B-ISI-A; the results were comparable and were therefore grouped together. (B) The training history of one subject. Each data point represents the performance of one session. The error bars show standard error of the mean (s.e.m). The $\mathrm{x}$-axis label indicates the stimulus ID (A vs. B or AB vs. B) or ISI (in ms) for each training session. All training sessions are plotted, including sessions 
when animals perform above chance ( $\mathrm{p}<0.01$; filled circle) and at chance (open circle). The performance varies with ISI, i.e. with task difficulty. (C) Rats learned to perform above chance at most ISIs on which they were trained. For each ISI, the bar represents the following ratio: (number of rats able to perform the task defined by this ISI above chance on at least one session)/(number of rats tested at this ISI during at least one session). The error bars show s.e.m. (D) Cumulative histogram showing the best performance session of all 15 rats trained on the ISI=5 ms task. (E) Performance declined with task difficulty within a subject. Performance is shown for sessions 5-10 for subject o (see also Fig. S2-o). The error bars show s.e.m. 